\title{
Statyba
}

\section{FINITE ELEMENTS FOR MODELLING BEAMS AFFECTED BY A DISTRIBUTED LOAD}

\section{S. Kalanta}

To cite this article: S. Kalanta (1999) FINITE ELEMENTS FOR MODELLING BEAMS AFFECTED

BY A DISTRIBUTED LOAD, Statyba, 5:2, 91-99, DOI: 10.1080/13921525.1999.10531442

To link to this article: https://doi.org/10.1080/13921525.1999.10531442

册 Published online: 26 Jul 2012.

Submit your article to this journal 2

山 Article views: 52 


\section{КОНЕЧНЫЕ ЭЛЕМЕНТЫ ПРИМЕНИТЕЛЬНО К СТЕРЖНЯМ ПОД ДЕЙСТВИЕМ РАСПРЕДЕЛЕННЫХ НАГРУЗОК}

\section{С. Каланта}

\section{1. Введение}

В многочисленных разработках по методу конечных элементов и исследованиях напряженно-деформированного состояния (НДС) стержневых конструкций цЈя описания изгиба стержней применяется конечный элемент с кубическим распределением прогибов [1-3]. Изгиб элемента в одной плоскости описывается четырьмя узловыми перемещениями: прогибами и угловыми перемещениями концевых сечений элемента. Однако изогнутая ось стержня, нагруженного поперечной равномерно распределенной нагрузкой, описывается функцией четвертой степени. Таким элементом нельзя непосредственно определить прогиб среднего сечения изгибаемого стержня, например, рителя рамы, который чаше всего интересует проектировщика. Прогиб среднего, как и любого другого промежуточного сечения, можно определить в виде суммы двух значений: 1) перемещения от узловых воздействий; опреде-ляется при помощи функшии интерполищи пере-мещений элемента; 2) перемещения жесткоза-крепленного стержня от местных нагрузок; опре-деляется аналитическим решением. Однако рядовой инженер-проектировщик не владеет таким способом расчета перемещений. Разработанный в [4] равновесный элемент второго порядка позво-ляет, кроме концевых перемещений, определять и интеграшьный прогиб изогнутого стержня, с помощью которого в дальнейшем можно опре-делить и действительное значение прогиба сред-него сечения. Однако при этом требуются допол-нителыные вычисления.

Для расчета прогиба среднего сечения стержень зачастую моделируется двумя элементами, соединенными в среднем сечении, что приводит К увеличению числа неизвестных и объема обрабатываемой информации. Стержни с нагрузкой, распределенной по линейному закону, прихо- дится моделировать еще большим числом конечных элементов.

В задачах оптимизации упруго-пластических конструкций с ограничениями перемещений необходимо, чтобы ограничиваемые перемещения выступали в качестве неизвестных явного вида. Поэтому, чтобы ограничить перемещения среднего сечения стержня, необходимо применять конечный элемент с дополнительной степенью свободы - прогибом среднего сечения.

В настоящей работе применительно $\mathrm{K}$ стержням, нагруженным равномерно и линейно распределенной нагрузкой, разрабатываются высокоточные конечные элементы с функцией прогибов четвертой и пятой степени соответственно. Их плоский изгиб описывается пятыю и шестыю узловыми перемещениями. Пятая и шестая степени свободы - прогиб и угловое перемешение среднего сечения элемента. $О$ возможности построения таких элементов сказано в [5]. Путем искпючения (конденсации) перемещений среднего узла элементы преобразуются в элементы с обычным числом степеней свободы. Получаются простые формулы расчета перемещений среднего сечения, удобные для применения рядовым проектировщиком. Поэтому предлагаемые элементы легко могут быть внедрены в инженерную практику. Они позволяют непосредственно определять прогиб среднего сечения и могут применяться в задачах оптимизации конструкций с ограничениями перемешений.

\section{2. Основные определения и матрица жесткости элемента}

При рассмотрении изгиба стержней будем пользоваться технической теорией изгиба балок. Это позволяет в дальнейшем вместо изгиба стержня рассматривать изгиб его оси. Вначале построим матрицу жесткости элемента, находя- 
щегося в состоянии плоского изгиба и растяжения-сжатия, применительно к стержню под действием равномерно распределенной нагрузки. Строится конешный элемент, узловые перемещения и силы которого показаны на рис.1.
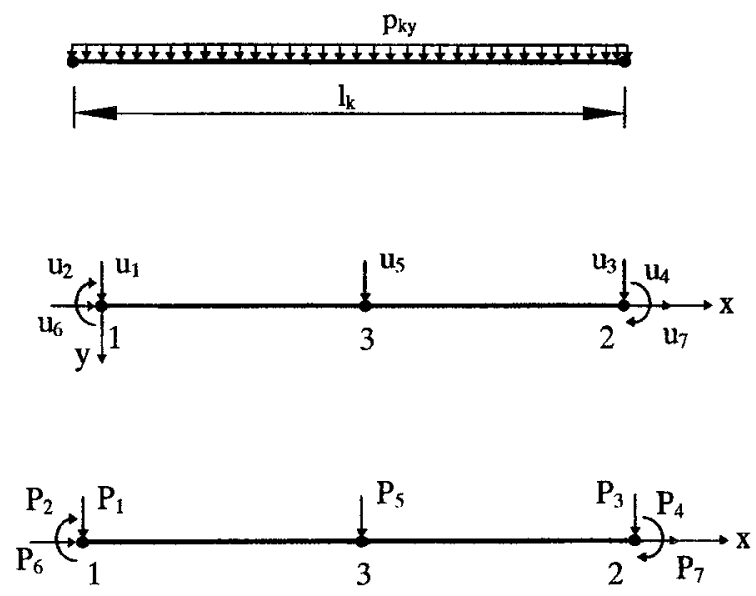

Рис. 1. Схема нагружения и положительные направления узловых перемешений и сил конечного элемента

Fig 1. Loading scheme and positive directions of node displacements and forces of a finite element

Положение элемента определяется семью узловыми перемещениями, т.е. вектором $\mathbf{u}_{k}=\left\{\mathbf{u}_{k l}, \mathbf{u}_{k l}\right\}^{T}=\left\{u_{k 1}, u_{k 2}, \ldots, u_{k 7}\right\}^{T}$. При этом первые пять перемещений $\mathbf{u}_{k l}=\left\{u_{k 1}, u_{k 2}, \ldots\right.$, $\left.u_{k 5}\right\}^{T}$ описывают изгиб элемента, а остальные растяжение-сжатие. Поэтому принимаются следующие законы распределения перемещений в элементе:

$$
\begin{aligned}
& u_{k y}(x)=a_{1}+a_{2} x+a_{3} x^{2}+a_{4} x^{3}+a_{5} x^{4}=\mathbf{h}_{k l}^{T}(x) \mathbf{a}_{k l} \\
& u_{k x}(x)=a_{6}+a_{7} x=\mathbf{h}_{k t}^{T}(x) \mathbf{a}_{k t},
\end{aligned}
$$

или

$$
\mathbf{u}_{k}(x)=\left[h_{k}(x)\right] \mathbf{a}_{k},
$$

где

$$
\begin{gathered}
\mathbf{u}_{k}(x)=\left\{u_{k y}(x), u_{k x}(x)\right\}^{T}, \quad \mathbf{a}_{k}=\left\{a_{1}, a_{2}, \ldots, a_{7}\right\}^{T}, \\
{\left[h_{k}(x)\right]=\left[\begin{array}{cc}
\mathbf{h}_{k l}^{T}(x) & 0 \\
0 & \mathbf{h}_{k t}^{T}(x)
\end{array}\right] .}
\end{gathered}
$$

При помощи функций (1) и граничных условий определяется связь

$$
\mathbf{u}_{k}=\left[H_{k}\right] \mathbf{a}_{k}
$$

и

$$
\mathbf{a}_{k}=\left[H_{k}\right]^{-1} \mathbf{u}_{k}
$$

Тогда функция перемешений (2) с уретом зависимости (4) принимает следующий вид:

$$
\mathbf{u}_{k}(x)=\left[H_{k}(x)\right] \mathbf{u}_{k}
$$

или

$$
\begin{gathered}
u_{k y}(x)=\sum_{i=1}^{5} H_{k i}(x) u_{k i}, \\
u_{k x}(x)=H_{k 6}(x) u_{k 6}+H_{k 7}(x) u_{k 7},
\end{gathered}
$$

где матрица функций формы

$$
\begin{aligned}
& {\left[H_{k}(x)\right]=\left[h_{k}(x)\right]\left[H_{k}\right]^{-1}=} \\
& {\left[\begin{array}{ccccccc}
H_{k 1}(x) & H_{k 2}(x) & H_{k 3}(x) & H_{k 4}(x) & H_{k 5}(x) & 0 & 0 \\
0 & 0 & 0 & 0 & 0 & H_{k 6}(x) H_{k 7}(x)
\end{array}\right]}
\end{aligned}
$$

$$
\begin{gathered}
H_{k 1}(x)=1-\frac{11 x^{2}}{l_{k}^{2}}+\frac{18 x^{3}}{l_{k}^{3}}-\frac{8 x^{4}}{l_{k}^{4}}, \\
H_{k 2}(x)=x-\frac{4 x^{2}}{l_{k}}+\frac{5 x^{3}}{l_{k}^{2}}-\frac{2 x^{4}}{l_{k}^{3}}, \\
H_{k 3}(x)=-\frac{5 x^{2}}{l_{k}^{2}}+\frac{14 x^{3}}{l_{k}^{3}}-\frac{8 x^{4}}{l_{k}^{4}}, \\
H_{k 4}(x)=\frac{x^{2}}{l_{k}}-\frac{3 x^{3}}{l_{k}^{2}}+\frac{2 x^{4}}{l_{k}^{3}}, \\
H_{k 5}(x)=\frac{16 x^{2}}{l_{k}^{2}}-\frac{32 x^{3}}{l_{k}^{3}}+\frac{16 x^{4}}{l_{k}^{4}}, \\
H_{k 6}(x)=1-\frac{x}{l_{k}},
\end{gathered}
$$

Kaк известно, матрица жесткости конечного элемента может быть построена с применением выражения потенциальной энергии элемента или на основе принципа возможных перемещений. С применением принципа возможных перемецений составляется уравнение равенства работ:

$$
\delta \mathbf{u}_{k}^{T} \mathbf{P}_{k}=\delta \mathbf{u}_{k}^{T} \int_{0}^{l}\left[B_{k}(x)\right]^{T}\left[d_{k}\right]\left[B_{k}(x)\right] \mathbf{u}_{k} d x,
$$

где

$$
\left[B_{k}(x)\right]=[\mathscr{A A}]^{T}\left[H_{k}(x)\right]
$$




$$
[\mathscr{A}]^{T}=\left[\begin{array}{cc}
-\frac{\partial^{2}}{\partial x^{2}} & 0 \\
0 & \frac{\partial}{\partial x}
\end{array}\right], \quad\left[d_{k}\right]=\left[\begin{array}{cc}
(E I)_{k} & 0 \\
0 & (E A)_{k}
\end{array}\right] .
$$

В силу произвольности вариаций $\delta \mathbf{u}_{k}$ получается уравнение жесткости конечного элемента:

$$
\mathbf{P}_{k}=\left[k_{k}\right] \mathbf{u}_{k},
$$

где матрица жесткости элемента

$$
\left[k_{k}\right]=\int_{0}^{l}\left[B_{k}(x)\right]^{T}\left[d_{k} \mathbf{I} B_{k}(x)\right] d x
$$

В нашем случае она состоит из двух частей матрицы жесткости изгиба $\left[k_{k l}\right]$ и матрицы жесткости растяжения-сжатия $\left[k_{k t}\right]$. Ее элементы вычисляются по формулам:

$$
k_{i j}=(E I)_{k} \int_{0}^{l} H_{k i}^{\prime \prime}(x) H_{k j}^{\prime \prime}(x) d x
$$

дия $i=1,2, \ldots, 5, \quad j=1,2, \ldots, 5$;

$$
k_{i j}=(E A)_{k} \int_{0}^{l} H_{k i}^{\prime}(x) H_{k j}^{\prime}(x) d x
$$

для $i=6,7, j=6,7$.

Здесь $H_{k i}^{\prime}(x), H_{k i}^{\prime \prime}(x)$ - первая и вторая производные функции формы перемещений $i$-го узла. В развернутой форме матрица жесткости элемента (рис. 1) представлена в табл. 1.
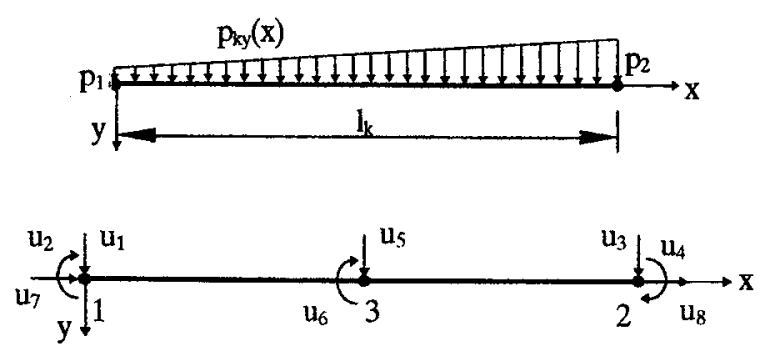

Рис. 2. Схема нагружения и узловые перемещения элемента

Fig 2. Loading scheme and node displacements of a finite element

Построим матрицу жесткости для элемента (рис. 2), находящегося в состоянии плоского изгиба и растяжения-сжатия, при линейно распределенной поперечной нагрузке. Интенсивность нагрузки описывается функцией:

$$
p_{k y}(x)=\left(1-\frac{x}{l_{k}}\right) p_{k 1}+\frac{x}{l_{k}} p_{k 2},
$$

где

$p_{k 1}, p_{k 2}$ - интенсивность нагрузки в узлах 1 и 3.

Изгиб элемента описывается дифференциальным уравнением:

$$
\frac{d^{4} u_{k y}(x)}{d x^{4}}=\frac{p_{k y}(x)}{(E I)_{k}},
$$

из которого следует, что его изогнутая ось описывается функцией пятой степени. Поэтому перемешения по длине элемента интерполируются полиномами

$$
\begin{aligned}
& u_{k y}(x)=a_{1}+a_{2} x+a_{3} x^{2}+a_{4} x^{3}+a_{5} x^{4}+a_{6} x^{5}, \\
& u_{k x}(x)=a_{7}+a_{8} x
\end{aligned}
$$

или

$$
\begin{aligned}
& u_{k y}(x)=\sum_{i=1}^{6} H_{k i}(x) u_{k i} \\
& u_{k x}(x)=H_{k 7}(x) u_{k 7}+H_{k 8}(x) u_{k 8},
\end{aligned}
$$

где

$$
\begin{aligned}
& H_{k 1}(x)=1-\frac{23 x^{2}}{l_{k}^{2}}+\frac{66 x^{3}}{l_{k}^{3}}-\frac{68 x^{4}}{l_{k}^{4}}+\frac{24 x^{5}}{l_{k}^{5}}, \\
& H_{k 2}(x)=x-\frac{6 x^{2}}{l_{k}}+\frac{13 x^{3}}{l_{k}^{2}}-\frac{12 x^{4}}{l_{k}^{3}}+\frac{4 x^{5}}{l_{k}^{4}}, \\
& H_{k 3}(x)=\frac{7 x^{2}}{l_{k}^{2}}-\frac{34 x^{3}}{l_{k}^{3}}+\frac{52 x^{4}}{l_{k}^{4}}-\frac{24 x^{5}}{l_{k}^{5}}, \\
& H_{k 4}(x)=-\frac{x^{2}}{l_{k}}+\frac{5 x^{3}}{l_{k}^{2}}-\frac{8 x^{4}}{l_{k}^{3}}+\frac{4 x^{5}}{l_{k}^{4}}, \\
& H_{k 5}(x)=\frac{16 x^{2}}{l_{k}^{2}}-\frac{32 x^{3}}{l_{k}^{3}}+\frac{16 x^{4}}{l_{k}^{4}}, \\
& H_{k 6}(x)=-\frac{8 x^{2}}{l_{k}}+\frac{32 x^{3}}{l_{k}^{2}}-\frac{40 x^{4}}{l_{k}^{3}}+\frac{16 x^{5}}{l_{k}^{4}}, \\
& H_{k 7}(x)=1-\frac{x}{l_{k}}, \quad H_{k 8}(x)=\frac{x}{l_{k}} .
\end{aligned}
$$

Коэффищиенты матрицы жесткости определяются по формулам (9) и (10). Матрица жесткости элемента с линейно распределенной нагрузкой приведена в табл. 2. 
Таблица 1. Матрица жесткости элемента с равномерно распределенной нагрузкой

Table 1. Stiffness matrix of a finite element affected by an evenly distributed load

\begin{tabular}{|c|c|c|c|c|c|c|c|}
\hline \multirow{7}{*}[\begin{array}{cc}{[k_{kl}]}&{[0]}\\
{[0]}&{[k_{kt}]}\end{array}]{$=$} & $316 E l / 5 l^{3}$ & $94 E I / 5 l^{2}$ & $196 E I / 5 l^{3}$ & $-34 E I / 5 l^{2}$ & $-512 E l / 5 l^{3}$ & & \\
\hline & $94 E I / 5 l^{2}$ & $36 E I / 5 l$ & $34 E I / 5 l^{2}$ & $-6 E I / 5 l$ & $-128 E I / 5 l^{2}$ & & \\
\hline & $196 E I / 5 l^{3}$ & $34 E I / 5 l^{2}$ & $316 E I / 5 l^{3}$ & $-94 E I / 5 l^{2}$ & $-512 E I / 5 l^{3}$ & & \\
\hline & $-34 E I / 5 l^{2}$ & $-6 E I / 5 l$ & $-94 E I / 5 l^{2}$ & $36 E I / 5 l$ & $128 E I / 5 l^{2}$ & & \\
\hline & $-512 E I / 5 l^{3}$ & $-128 E I / 5 l^{2}$ & $-512 E I / 5 l^{3}$ & $128 E I / 5 l^{2}$ & $1024 E I / 5 l^{3}$ & & \\
\hline & & & & & & $E A / l$ & $-E A / l$ \\
\hline & & & & & & $-E A / l$ & $E A / l$ \\
\hline
\end{tabular}

Таблица 2. Матрица жесткости элемента с линейно распределенной нагрузкой

Table 2. Stiffness matrix of a finite element affected by a straight distributed load

$\left[k_{k}\right]=$\begin{tabular}{|c|c|c|c|c|c|c|c|}
\hline $5092 E I / 35 l^{3}$ & $1138 E I / 35 l^{2}$ & $-1508 E I / 35 l^{3}$ & $242 E I / 35 l^{2}$ & $-512 E I / 5 l^{3}$ & $384 E I / 7 l^{2}$ & & \\
\hline $1138 E I / 35 l^{2}$ & $332 E I / 35 l$ & $-242 E I / 35 l^{2}$ & $38 E I / 35 l$ & $-128 E I / 5 l^{2}$ & $64 E I / 7 l$ & & \\
\hline$-1508 E I / 35 l^{3}$ & $-242 E I / 35 l^{2}$ & $5092 E I / 35 l^{3}$ & $-1138 E I / 35 l^{2}$ & $-512 E I / 5 l^{3}$ & $-384 E I / 7 l^{2}$ & & \\
\hline $242 E I / 35 l^{2}$ & $38 E I / 35 l$ & $-1138 E I / 35 l^{2}$ & $332 E I / 35 l$ & $128 E I / 5 l^{2}$ & $64 E I / 7 l$ & & \\
\hline$-512 E I / 5 l^{3}$ & $-128 E I / 5 l^{2}$ & $-512 E I / 5 l^{3}$ & $128 E I / 5 l^{2}$ & $1024 E I / 5 l^{3}$ & 0 & & \\
\hline $384 E I / 7 l^{2}$ & $64 E I / 7 l$ & $-384 E I / 7 l^{2}$ & $64 E I / 7 l$ & 0 & $256 E I / 7 l$ & & \\
\hline & & & & & & $E A / l$ & $-E A / l$ \\
\hline & & & & & & $-E A / l$ & $E A / l$ \\
\hline
\end{tabular}

Матрица жесткости элемента при совместном учете деформапий изгиба, растяжениясжатия и кручения состоит из четырех матриц жесткости, расположенных по диагонали:

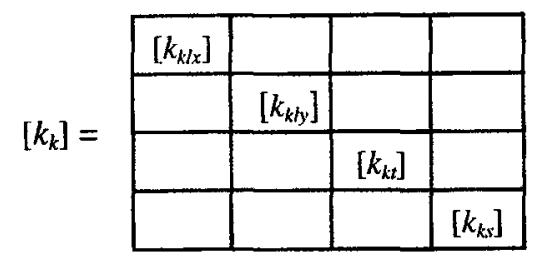

Здесь $\left[k_{k l x}\right],\left[k_{k l y}\right]$ - матрицы жесткости изгиба в плоскостях $x z$ и $y z$ - определены согласно (9); [ $\left.k_{k t}\right]$ - матрица жесткости растяжения-сжатия определена согласно (10); $\left[k_{k s}\right] \quad$ - матрица жесткости кручения - определена по формуле:

$$
\left[k_{k s}\right]=\frac{\left(G I_{s}\right)_{k}}{l_{k}}\left[\begin{array}{rr}
1 & -1 \\
-1 & 1
\end{array}\right],
$$

где $G I_{s}$ - жесткость стержня на кручение.

\section{3. Узловые силы элемента}

Вектор узловых сил, эквивалентных распределенной нагрузке элемента, определяется из условия равенства работ узловых сил и распределенной нагрузки:

$$
\mathbf{u}_{k}^{T} \mathbf{F}_{k}=\int_{0}^{l} \mathbf{u}_{k}^{T}(x) \mathbf{p}_{k}(x) d x,
$$

где $\mathbf{p}_{k}(x)=\left\{p_{k y}(x), p_{k x}(x)\right\}^{T}$ - вектор интенсивности распределенных на элементе нагрузок. Отсюда вектор узловых сил с учетом (5)

$$
\mathbf{F}_{k}=\int_{0}^{l}\left[H_{k}(x)\right]^{T} \mathbf{p}_{k}(x) d x .
$$

При условиях $\quad p_{k x}(x)=p_{k x}=$ const, $p_{k y}(x)=p_{k y}$ узловые силы конечного элемента, показанного на рис. 1, определяются вектором 


$$
\begin{aligned}
\mathbf{F}_{k}= & \left\{\frac{7}{30} p_{k y} l_{k}, \frac{1}{60} p_{k y} l_{k}^{2}, \frac{7}{30} p_{k y} l_{k},-\frac{1}{60} p_{k y} l_{k}^{2},\right. \\
& \left.\frac{16}{30} p_{k y} l_{k}, \frac{1}{2} p_{k x} l_{k}, \frac{1}{2} p_{k x} l_{k}\right\}^{T} .
\end{aligned}
$$

На рис.3 показаны узловые сишы этого элемента, a также элемента с линейно распределенной поперечной нагрузкой. Очевидно, что последний элемент (рис. 2) можно применять и для моделирования стержней с равномерно распределенной нагрузкой.

\section{4. Редуцированные элементы и их применение}

Представленные конешные элементы имеют внутренние степени свободы. Их можно выразить через внешние степени - перемещения конечных узлов элемента и в дальнейшем исключить из числа неизвестных задачи. При этом соответст- венно преобразуются матрица жесткости и вектор узловых сил элемента.

Для этой цели узловые перемещения и силы элемента разделяем на внутренние $\mathbf{u}_{k v}, \mathbf{P}_{k v}$ и внешние $\mathbf{u}_{k s}, \mathbf{P}_{k s}$. Соответственно матрица жесткости разделяется на 4 подматрицы:

$$
\left[k_{k}\right]=\left[\begin{array}{ll}
{\left[k_{s s}\right]} & {\left[k_{s v}\right]} \\
{\left[k_{v s}\right]} & {\left[k_{v v}\right]}
\end{array}\right] .
$$

Тогда уравнение жесткости (7) можно разделить на следующие две группы:

$$
\begin{aligned}
& \mathbf{P}_{k s}=\left[k_{s s}\right] \mathbf{u}_{k s}+\left[k_{s v}\right] \mathbf{u}_{k v}, \\
& \mathbf{P}_{k v}=\left[k_{v s}\right] \mathbf{u}_{k s}+\left[k_{v v}\right] \mathbf{u}_{k v} .
\end{aligned}
$$

Отсюда внутренние степени свободы

$$
\mathbf{u}_{k v}=\left[k_{v v}\right]^{-1} \mathbf{P}_{k v}-\left[k_{v v}\right]^{-1}\left[k_{v s}\right] \mathbf{u}_{k s} .
$$

a)

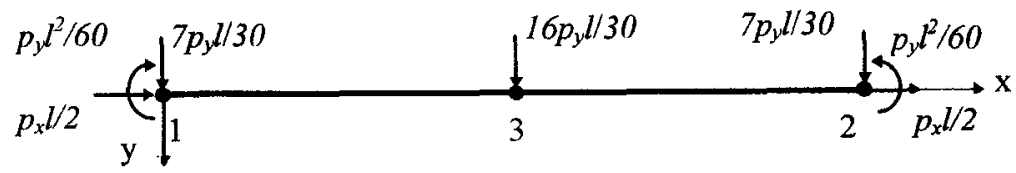

б)

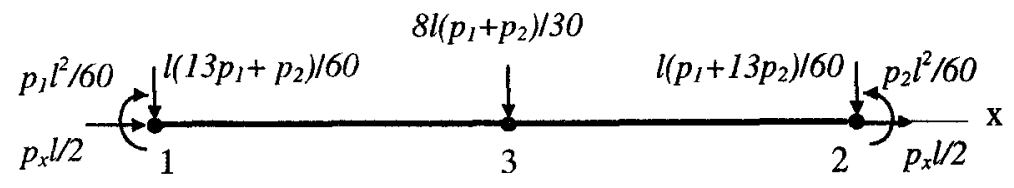

B)

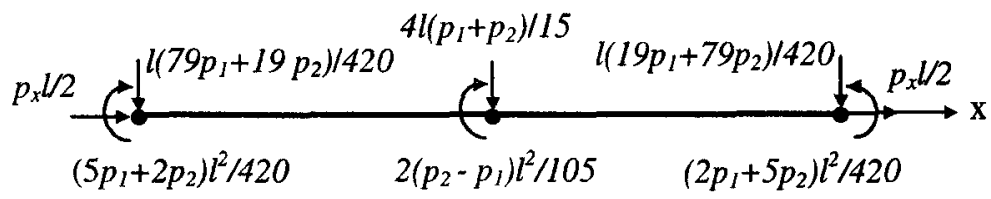

Рис. 3. Узловые силы конечных элементов:

а) элемента с функпией прогибов четвертой степени при равномерно распределенной нагрузке;

б) элемента с фупкцией прогибов четвертой степени при линейно распределенной нагрузке;

в) элемента с функцией прогибов пятой степени при линейно распределенной нагрузке

Fig 3. Node forces of finite elements:

a) of the element with fourth-order deflection approximation function and evenly distributed load;

б) of the element with fourth-order deflection approximation function and straight distributed load;

B) of the element with fifth-order deflection approximation function and straight distributed load

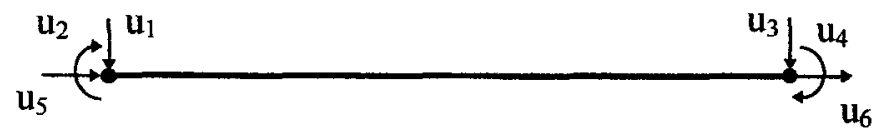

Рис. 4. Узловые перемещения редуцированного элемента

Fig 4. Node displacements of a reduced finite element 
a)

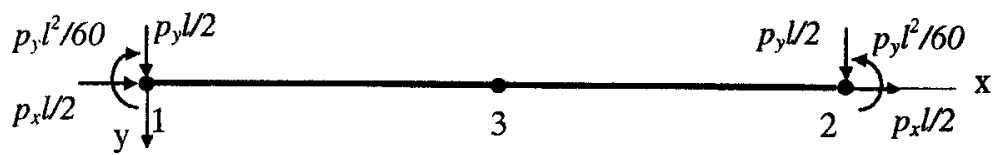

б)

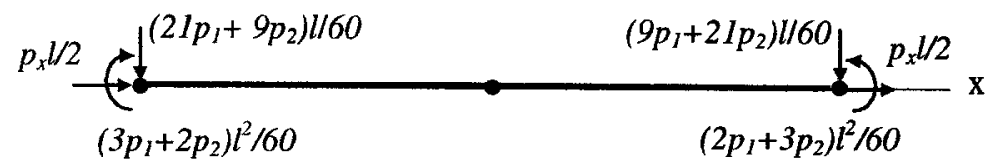

Рис. 5. Узловые силы редуцированного элемента:

a) при равномерно распределенной нагрузке;

б) при линейно распределенной нагрузке

Fig 5. Node forces of reduced finite element: a) for an evenly distributed load; $b$ ) for a straight distributed load

Подставляя это выражение в первое уравнение системы (13), получаем преобразованное уравнение жесткости элемента:

$$
\widetilde{\mathbf{P}}_{k}=\left[\widetilde{k}_{k}\right] \widetilde{\mathbf{u}}_{k},
$$

где $\widetilde{\mathbf{u}}_{k} \equiv \mathbf{u}_{k s}$. Преобразованная матрица жесткости

$$
\left[\tilde{k}_{k}\right]=\left[k_{s s}\right]-\left[k_{s v}\right]\left[k_{v v}\right]^{-1}\left[k_{v s}\right]
$$

и вектор обобщенных сил

$$
\tilde{\mathbf{P}}_{k}=\mathbf{P}_{k s}-\left[k_{s v}\right]\left[k_{v v}\right]^{-1} \mathbf{P}_{k v} .
$$

Построим матрицы жесткости и векторы внешних узловых сил редуцированных конечных элементов. На рис. 4 показаны узловые перемещения этих элементов. Внешние узловые силы редуцированного элемента определяются по формуле:

$$
\tilde{\mathbf{F}}_{k}=\mathbf{F}_{k s}-\left[k_{s v}\right]\left[k_{v v}\right]^{-1} \mathbf{F}_{k v} .
$$

Они показаны на рис. 5. При исключении внутренних степеней свободы представленных

\begin{tabular}{|c|c|c|c|c|}
\hline \multirow{4}{*}[k_{k}]{$=E I$} & $12 / t^{3}$ & $6 /^{2}$ & $-12 h^{3}$ & $6 l^{2}$ \\
\hline & $6 / l^{2}$ & $4 h$ & $-6 / l^{2}$ & $2 / l$ \\
\hline & $-12 / t^{3}$ & $-6 / l^{2}$ & $12 /^{3}$ & $.6 h^{2}$ \\
\hline & $6 / l^{2}$ & $2 / l$ & $-6 / l^{2}$ & $4 / /$ \\
\hline
\end{tabular}
элементов изменяются тољко матрицы жесткости изгиба. Поэтому ниже приводится лишь редуцированная матрица жесткости изгиба:
Она одинакова как для элементов с равномерно, так и с линейно распределенными нагрузками.

Зависимость (15) позволяет определять перемещения внутренних узлов элементов уже после решения системы разрешающих уравнений конечноэлементной модели. Используя зависимость (15), а также приведенные выше матрицы жесткости и узловые силы, получаем следующиие прикладные формулы для расчета перемещений внутренних узлов:

a) при равномерно распределенной нагрузке-

$u_{5}=\frac{p_{y} l^{4}}{384 E I}+0.5 u_{1}+0.125 l u_{2}+0.5 u_{3}-0.125 l u_{4}$,

б) при линейно распределенной нагрузке -

$$
\begin{aligned}
& u_{5}=\frac{\left(p_{1}+p_{2}\right) l^{4}}{768 E I}+0.5 u_{1}+0.125 l u_{2}+0.5 u_{3}-0.125 l u_{4}, \\
& u_{6}=\frac{\left(p_{2}-p_{1}\right) l^{3}}{1920 E I}-\frac{15}{l} u_{1}-0.25 u_{2}+\frac{15}{l} u_{3}-0.25 u_{4} .
\end{aligned}
$$

Используя эти формулы, можно исключить перемещения среднего узла элемента и преобразовать функции перемещений (6), (13) в следующие формы:

$$
u_{k y}(x)=\tilde{u}_{k y}(x)+\frac{p_{k y} l_{k}^{4}}{24(E I)_{k}}\left(\frac{x^{2}}{l_{k}^{2}}-\frac{2 x^{3}}{l_{k}^{3}}+\frac{x^{4}}{l_{k}^{4}}\right)
$$




$$
\begin{array}{r}
u_{k y}(x)=\tilde{u}_{k y}(x)+\frac{\left(p_{1}+p_{2}\right) l_{k}^{4}}{48(E I)_{k}}\left(\frac{x^{2}}{l_{k}^{2}}-\frac{2 x^{3}}{l_{k}^{3}}+\frac{x^{4}}{l_{k}^{4}}\right)+ \\
+\frac{\left(p_{2}-p_{1}\right) l_{k}^{4}}{240(E I)_{k}}\left(-\frac{x^{2}}{l_{k}^{2}}+\frac{4 x^{3}}{l_{k}^{3}}-\frac{5 x^{4}}{l_{k}^{4}}+\frac{2 x^{5}}{l_{k}^{5}}\right)
\end{array}
$$

где

$$
\begin{aligned}
\tilde{u}_{k y}(x) & =\left(1-\frac{3 x^{2}}{l_{k}^{2}}+\frac{2 x^{3}}{l_{k}^{3}}\right) u_{k 1}+\left(x-\frac{2 x^{2}}{l_{k}}+\frac{x^{3}}{l_{k}^{2}}\right) u_{k 2}+ \\
& +\left(\frac{3 x^{2}}{l_{k}^{2}}-\frac{2 x^{3}}{l_{k}^{3}}\right) u_{k 3}+\left(-\frac{x^{2}}{l_{k}}+\frac{x^{3}}{l_{k}^{2}}\right) u_{k 4},
\end{aligned}
$$

$\tilde{u}_{k y}(x)$ - интерполирующая функция прогибов обычного двухузлового элемента. Второй член зависимости (22) выражает погрешность прогиба сечений стержня, когда он моделируется одним обычным конечным элементом.

Зависимости (22) и (23) также получаются интегрированием дифференциального уравнения (12) с учетом граничных условий:

$$
\begin{gathered}
\left.u_{k y}(x)\right|_{x=0}=u_{k 1},\left.u_{k y}(x)\right|_{x=l}=u_{k 3},\left.u_{k y}(x)\right|_{x=0.5 l}=u_{k 5}, \\
\left.\frac{d u_{k y}(x)}{d x}\right|_{x=0}=u_{k 2},\left.\frac{d u_{k y}(x)}{d x}\right|_{x=l}=u_{k 4}
\end{gathered}
$$

И

$$
\left.\frac{d u_{k y}(x)}{d x}\right|_{x=0.5 l}=u_{k 6} .
$$

Шестое условие учитывается только для элемента $\mathrm{c}$ линейно распределенной нагрузкой. Таким образом, зависимости (22) и (23) являются аналитическим решением дифференциалного уравнения (12). Это свидетельствует о том, что представленные конеqные элементы совершенно точно моделируют напряженно-деформированное состояние изгибаемых стержней.

Матрица жесткости редуцированных элементов совпадает с матрищей жесткости обычно используемого элемента с кубическим распределением прогибов. Узловые силы редуцированного элемента с равномерно распределенной нагрузкой также равны силам обычного элемента. Поэтому для расчета стержневых конструкций при равномерно распределенной нагрузке можно использовать широко распространенные программы, в которых плоский изгиб стержня моделируется четырьмя узловыми перемещениями. Для расчета прогиба среднего сечения стержня целесообразно применять формулу (20), которая может быть включена в программы. Эти программы можно испољзовать и для анализа стержней с линейно распределенной нагрузкой, однако для расчета узловых сил конечных элементов следует применять формулы, показанные на рис. 3 в, а для расчета перемещений средних сечений - формулы (21). При этом каждый стержень достаточно моделировать одним конечным элементом, если жесткость постоянная.

\section{5. Расчет усилий стержней}

Усилия в стержнях, подверженных действию местной нагрузки, состоят из двух частей:

- усилия от перемещений его узлов, которые определяются по формуле (7);

- усилия от местной нагрузки.

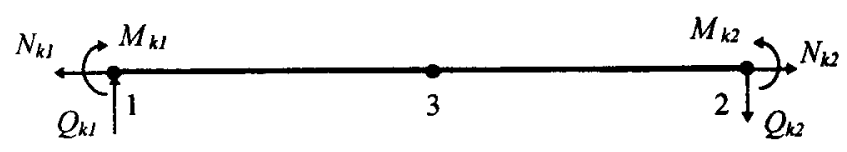

Рис. 6. Положительные направления усилий

Fig 6. Positive directions of stresses

Пусть положительные направления усилий опре-деляются согласно рис. 6. Тогда изгибающие моменты и поперечные силы в концах элемента определяются зависимостями:

$$
\begin{array}{ll}
Q_{k 1}=-P_{k 1}+F_{k 1}, & M_{k 1}=P_{k 2}-F_{k 2}, \\
Q_{k 2}=P_{k 3}-F_{k 3}, & M_{k 2}=-P_{k 4}+F_{k 4} .
\end{array}
$$

Продольные силы

и

$$
\begin{array}{ll}
N_{k 1}=-P_{k 6}+F_{k 6}, & N_{k 2}=P_{k 7}-F_{k 7} \\
N_{k 1}=-P_{k 7}+F_{k 7}, & N_{k 2}=P_{k 8}-F_{k 8} .
\end{array}
$$

Изгибаюший момент в третьем узле

$$
\begin{aligned}
& M_{k 3}=\left(M_{k 1}+M_{k 2}\right) / 2+p_{k y} l_{k}^{2} / 8 . \\
& M_{k 3}=\frac{(E I)_{k}}{l_{k}}\left(u_{2}-u_{4}\right)+\frac{\left(p_{1}+p_{2}\right) l_{k}^{2}}{48} .
\end{aligned}
$$

С использованием зависимостей

$M_{k}(x)=-(E I)_{k} \frac{d^{2} u_{k}(x)}{d x^{2}}, \quad Q_{k}(x)=-(E I)_{k} \frac{d^{3} u_{k}(x)}{d x^{3}}$

получаются следующие формулы для расчета усилий в лобом промежуточном сечении элемента: 
a) при равномерно распределенной нагрузке -

$$
\begin{aligned}
M_{k}(x)= & \frac{(E I)_{k}}{l_{k}}\left[\left(\frac{6}{l_{k}}-\frac{12 x}{l_{k}^{2}}\right) u_{k 1}+\left(4-\frac{6 x}{l_{k}}\right) u_{k 2}+\right. \\
& \left.+\left(-\frac{6}{l_{k}}+\frac{12 x}{l_{k}^{2}}\right) u_{k 3}+\left(2-\frac{6 x}{l_{k}}\right) u_{k 4}\right]+ \\
& +\frac{p_{k y} l_{k}^{2}}{12}\left(-1+\frac{6 x}{l_{k}}-\frac{6 x^{2}}{l_{k}^{2}}\right), \\
Q_{k}(x)= & \frac{(E I)_{k}}{l_{k}^{2}}\left(-\frac{12}{l_{k}} u_{k 1}-6 u_{k 2}+\frac{12}{l_{k}} u_{k 3}-6 u_{k 4}\right)+ \\
& +\frac{p_{k y} l_{k}}{2}\left(1-\frac{2 x}{l_{k}}\right)
\end{aligned}
$$

б) при линейно распределенной нагрузке -

$$
\begin{aligned}
& M_{k}(x)= \frac{(E I)_{k}}{l_{k}}\left[\left(\frac{6}{l_{k}}-\frac{12 x}{l_{k}^{2}}\right) u_{k 1}+\left(4-\frac{6 x}{l_{k}}\right) u_{k 2}+\right. \\
&\left.+\left(-\frac{6}{l_{k}}+\frac{12 x}{l_{k}^{2}}\right) u_{k 3}+\left(2-\frac{6 x}{l_{k}}\right) u_{k 4}\right]+ \\
&+\frac{\left(p_{1}+p_{2}\right) l_{k}^{2}}{24}\left(-1+\frac{6 x}{l_{k}}-\frac{6 x^{2}}{l_{k}^{2}}\right)+ \\
&+\frac{\left(p_{2}-p_{1}\right) l_{k}^{2}}{120}\left(1-\frac{12 x}{l_{k}}+\frac{30 x^{2}}{l_{k}^{2}}-\frac{20 x^{3}}{l_{k}^{3}}\right) \\
& Q_{k}(x)= \frac{(E I)_{k}}{l_{k}^{2}}\left(-\frac{12}{l_{k}} u_{k 1}-6 u_{k 2}+\frac{12}{l_{k}} u_{k 3}-6 u_{k 4}\right)+ \\
&+\frac{\left(p_{1}+p_{2}\right) l_{k}}{4}\left(1-\frac{2 x}{l_{k}}\right)+\frac{\left(p_{2}-p_{1}\right) l_{k}}{10}\left(-1+\frac{5 x}{l_{k}}-\frac{5 x^{2}}{l_{k}^{2}}\right) .
\end{aligned}
$$

\section{6. Выводы}

Построены новые конечные элементы с фунхциями прогибов четвертой и пятой степени применительно к изгибаемым стержням под действием распределенных нагрузок. В рамках технической теории изгиба балок они позволяют моделировать напряженно-деформированное состояние стержня при помощи одного конечного элемента. Кроме того, они позволяют непосредственно определить перемешения среднего сечения элемента. Это позволяет уменьшить объем задачи и выводимой информации, которую необходимо обработать после решения задачи.
Путем исключения внутренних степеней свободы построены редуцированные конечные элементы, число степеней свободы которых уменышено до числа степеней свободы обычного (двухузлового) элемента. При этом предложенные редуцированные элементы сохраняют вышеуказанные преимущества. Получены простые формулы (20) и (21), при помощи которых перемещения среднего узла элемента вырахаются через перемещения концевых узлов. Они позволяют определять перемещения среднего сечения стержня уже после решения системы алгебраических уравнений и удобны для применения рядовым проектировщиком. Редуцированные элементы легко могут быть внедрены в инженерную практику, так как их матрищы жесткости совпадают с матрицей жесткости обычного стержневого элемента.

Конечные элементы с внутренними степенями свободы важны для задач оптимизации упруго-пластических конструкщий с ограничениями перемещений, так как для обеспечения ограничения перемещения среднего сечения стержня необходимо, чтобы это перемещение присутствовало в качестве неизвестного в явном виде. При этом крайне вахно уменышить число неизвестных задачи оптимизации.

\section{Литература}

1. В. А. Постнов, И. Я. Хархурим. Метод конечньх элементов в расчетах судовых конструкций. Ленинград: Судостроение, 1974. $342 \mathrm{c}$.

2. О. Зенкевич. Метод конечных элементов в технике. М: Мир, 1975. 541 c.

3. Р. Галлагер. Метод конечных элементов. Основы. M: Мup, 1984. 428 c.

4. С. А. Каланта. Упругий расчет плоских стержневых систем методом конечных элементов // Литовский механический сборник: Вопросы теоретической и прикладной механики, № 26, 1983, с. 78-94.

5. R. Kačianauskas. Computer methods in multilevel modelling of beams and shells. Vilnius: Technika, 1995. 395 p.

Iteikta 19990423

\section{BAIGTINIAI ELEMENTAI STRYPAMS SU PASKIRSTYTOMIS APKROVOMIS MODELIUOTI}

\section{S. Kalanta}

\section{Santrauka}

Lenkiamu strypu analizei paprastai naudojami baigtiniai elementai su kubiniu jlinkiu pasiskirstymu.

Straipsnyje strypams, veikiamiems paskirstytuju apkrovų, modeliuoti sudaryti baigtiniai elementai $(1,2$ pav.) su 
ketvirtojo ir penktojo laipsnio jlinkiu interpoliavimo funkcijomis (1), (6) ir (13). Penktojo laipsnio jlinkiu funkcijos naudojamos strypams su tiesiškai paskirstyta apkrova (11) modeliuoti. Elemento ploǩčiasis lenkimas modeliuojamas 5 ir 6 laisvumo laipsniais. Papildomieji 5 ir 6 laisvumo laipsniai - elemento viduriniojo mazgo 3 ilinkis ir kampinis poslinkis (2 pav.). Sudarytos elementy standumo matricos (1, 2 lent.) ir išorinių jègų vektoriai. Sudaryti baigtiniai elementai tiksliai modeliuoja paskirstytosiomis apkrovomis veikiamų strypu ittempimų ir deformacijų būvị, be to, leidžia tiesiogiai skaičiuoti strypy viduriniojo pjūvio poslinkius. Visa tai sudaro galimybę sumažinti uždavinių ir gaunamos informacijos, kurią vèliau reikia analizuoti, apimtị.

Eliminuojant vidinius laisvumo laipsnius, sudaryti redukuotieji baigtiniai elementai (4 pav.), kuriu laisvumo laipsnių skaičius sumažintas iki paprastai naudojamo elemento laisvumo laipsnių skaičiaus. Tačiau redukuotieji elementai turi visus nurodytuosius privalumus. Gautos formulès (20) ir (21), kuriomis elemento viduriniojo mazgo poslinkiai išreiškiami per jo galiniu mazgu poslinkius. Jos leidžia apskaičiuoti strypo viduriniojo pjūvio poslinkius jau po baigtiniu elementų lygčių sistemos sprendimo.

Pasiūlyti redukuotieji elementai gali būti labai lengvai įdiegti ir naudojami inžinerinèje praktikoje, kadangi ju standumo matrica sutampa su iprastinio strypinio elemento standumo matrica.

Sudaryti elementai su vidiniais laisvumo laipsniais ypač svarbūs konstrukcijų optimizacijos su poslinkių ribojimais uždaviniams, nes, norint apriboti strypo viduriniojo pjūvio poslinkị, reikia, kad šis poslinkis būtł uždavinio nežinomasis. Be to, labai svarbu kiek imanoma sumažinti optimizacijos uždavinio nežinomųju skaičių.

\section{FINITE ELEMENTS FOR MODELLING BEAMS AFFECTED BY A DISTRIBUTED LOAD}

\section{S. Kalanta}

\section{S u m m ary}

Usually a finite element with cubic deflection approximation function is applied when evaluating the stress and strain field of bar structures. But such an element only approximately evaluates the actual strain field of the bar affected by a distributed load. The improved finite elements
(Fig 1,2) with fourth and fifth-order deflection approximation functions (1), (6) and (13) are presented in the actual manuscript. The fifth-order deflection approximation function is used for modelling the beams affected by a linearly distributed load (11). The plain bending of the finite element is modelled by 5 and 6 freedom degrees. The additional $5^{\text {th }}$ and $6^{\text {th }}$ freedom degrees are the deflection and deviation of the middle node of element (Fig 2). The element stiffness matrices (Table 1,2 ) and node force vectors are presented. The created finite elements exactly modells the stress and strain field of bars, which are affected by distributed load, and also allow to compute directly the middle section displacements of bars. It creates conditions for diminishing the volume of problems and obtaining information, which is necessary to be analysed later.

The reduced finite elements (Fig 4) are created by the elimination of the internal freedom degrees. Their number of freedom degrees is decreased up to the number of freedom degrees of a usually applied finite element. But the reduced finite elements have all afore-mentioned qualities. Formulas (20) and (21) are derived expressing the middle node displacements by the final node displacements. These formulas allow to compute the middle section displacements of the bar already after the solution of equation system.

The proposed reduced elements can be introduced and applied in engineering practice very easily, because their stiffness matrix coincide with the stiffness matrix of a usual bar finite element

The created elements with internal freedom degrees are very important for the problems of structures optimization with displacement constraints, because the constraint of bar middle section displacement can form just in case, when this displacement is one of the problem's unknown. Also it is very important to decrease the number of unknowns of optimization problem.

Stanislovas KALANTA. Doctor, Associate Professor. Dept of Structural Mechanics. Vilnius Gediminas Technical University, Sauletekio al. 11, 2040 Vilnius, Lithuania.

Doctor (structural mechanics), 1974. Research visits to: Leningrad Polytechnic Institute, Moscow Civil Engineering Institute, Kiev Civil Engineering Institute. Research interests: computational mechanics, finite element method, analysis and optimization of elastic-plastic structures. 